\title{
La radionovela como práctica pedagógica para el aprendizaje de lectura en básica primaria

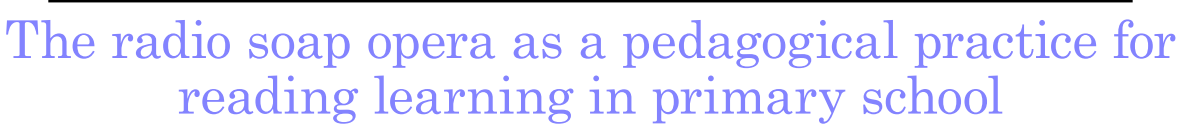

DOI: http://dx.doi.org/10.17981/cultedusoc.9.3.2018.76

Artículo de investigación. Fecha de recepción: 15/06/2018. Fecha de aceptación: 27/11/2018

\author{
Adalberto Alonso Munive - Pacheco; Adela Ines Carbono - Parejo; Cesar Camargo \\ - Cervantes; Dannis Peralta - Angulo; Dubis Montenegro - Castro; John Imitola \\ - Guerra; Leysle Polo - Carrillo; Marlene Varela - Ávila; Omaira Crespo - Ponce; \\ Piedad Hernández - Miranda; Shirley Candelario - Martínez; Víctor Gómez - Tovar; \\ Yeisly Mendoza - Oñate y Sobeida Santrich - Angulo1
}

Para citar este artículo:

Perea-Hernández, A., Colina-Páez, A., Vargas-Guerrero, B., Carrillo-Álvarez, D., Meriño-Bermúdez, D., Bolaño-Hernández, D., Olarte-Faillace, E., Castro-Hernández, G., Rodríguez-Zúñiga, G., Gámez-Lobo, H., Padilla-Jiménez, K., Padilla-Jiménez, L. Martínez-Ordoñez, L., Charris-Matute, L., Ibarra-Orozco, L., Arévalo-Fandiño, M., Rodríguez-Hernández, M., Gutiérrez-Daza, M., Villa-Carmona, O., Núñez-Anaya, R., Pérez-Cervantes, S., Navarro-Montenegro, S. y Duran-Varela, Y. (2018). Lectura y escritura a través de la investigación como estrategia pedagógica apoyada en las TIC. Cultura. Educación y Sociedad 9(3), 645-654. DOI: http:// dx.doi.org/10.17981/cultedusoc.9.3.2018.76

\section{Resumen}

La radionovela es un género radial en desuso con un lenguaje coloquial que conmueve a los oyentes en sus conductas lingüísticas a través de su influencia cotidiana. Cuyo lenguaje puede favorecer el aprendizaje en la comprensión lectora de los estudiantes. El estudio tuvo como propósito implementar la radionovela como práctica pedagógica para el aprendizaje de lectura en básica primaria. Este estuvo guiado bajo los lineamientos metodológicos de la IEP con un diseño basado en las trayectorias de indagación. Se utilizaron técnicas de recolección de datos como; la observación participante y el diario de campo. La población estuvo conformada por cuarenta (40) estudiantes de básica primaria de la IED Cerro Blanco del Municipio de Zona Bananera, Magdalena. Se evidencio que los estudiantes mejoraron su comprensión lectora y por ende su desempeño académico observando que el acompañamiento en casa muchas veces era limitado porque los adultos no sabían leer y a través de la implementación de la radionovela lograron comprender y acompañar a los estudiantes en el proceso de aprendizaje, concluyendo que cualquier acción educativa está compuesta por relaciones sociales, están manifiestan bajo formas pedagógicas aquellas representaciones sociales que son visibles en este género radial.

Palabras clave: radionovela, practica pedagógica, lectura.

\section{Abstract}

The radionovela is a disused radio genre with a colloquial language that moves the listeners in their linguistic behaviors through their daily influence. Whose language can favor learning in the reading comprehension of students. The purpose of the study was to implement the radionovela as a pedagogical practice for reading learning in elementary school. This was guided under the methodological guidelines of the IEP with a design based on the trajectories of inquiry. Data collection techniques were used, such as; the participant observation and the field diary. The population was conformed by forty (40) primary students of the IED Cerro Blanco of the Municipality of Zona Bananera, Magdalena. It was evidenced that the students improved their reading comprehension and therefore their academic performance observing that the accompaniment at home was often limited because the adults did not know how to read and through the implementation of the radionovela they were able to understand and accompany the students in the process of learning, concluding that any educational action is composed of social relations, are manifested under pedagogical forms those social representations that are visible in this radial genre.

Keywords: radionovela, pedagogical practice, reading.

1 Docentes de la IED Cerro Blanco, sede principal.

- The author; licensee Universidad de la Costa - CUC.

Cultura, Educación y Sociedad vol. 9 no. 3, pp. 645-654. Diciembre, 2018

Barranquilla. ISSN 2389-7724 Online 


\section{Introducción}

La radionovela comenzó a transmitirse por las ondas radiales a principios del siglo XX. Al igual que los escritores televisivos y cinematográficos, los libretistas radiofónicos conocen muy bien las expectativas del oyente. Desde sus orígenes, el radio-drama fue adoptando los disfraces que le han servido hasta nuestros días. Los países latinoamericanos, entre ellos México, han sufrido a lo largo de su historia diversos conflictos económicos, políticos y sociales.

En la actualidad, el género tele -novelesco de la televisión ha tomado tanta fuerza en las comunicaciones que ha reemplazado todas las formas anteriores de cotidianidad existentes con anterioridad en la interacción con los miembros de las familias, como pudieron ser los relatos de los abuelos y padres o los programas radiales en su tiempo. Este medio comparte un espacio con el internet y las redes sociales, que en su contexto influyen en las conductas lingüísticas de los receptores (Cisneros, Olave y Rojas, 2013). "La novela televisiva en sus años de historia es de las pocas que impacta tanto a la sociedad por reunir a la familia en torno al televisor" (Cisneros, 2003, p. 126). Transmitiendo a través de sus historias no solo sentimientos sino el voyeurismo al entrar en contacto con la intimidad de los personajes que las cuentan (Vilches, 1997).

$\mathrm{Su}$ antecesora, la radionovela nace en los años 30 con la transmisión de "La guerra de los mundos" con un gran impacto por el realismo de su dramatización. Con un éxito que en la actualidad todavía se mantiene en la preferencia del público de esa época, por ser un formato que atraía a una gran audiencia y gran cantidad de patrocinadores del formato "soap opera" con que era conocida por esos años (Trujillo, 2013). A finales de los años 60 la televisión desplaza este medio con grandes éxitos que todavía se conservan en la actualidad, pasando a un plano más musical, conservando hasta hoy este formato, como Haye (1995) comenta:

"Lo lamentable de la cuestión es que la radio, se fue acostumbrando a su papel de oferente pobre y también acostumbró a las nuevas generaciones a esperar de ella ese mezquino tríptico de música- noticias- tandas comerciales" (p. 181).

Los jóvenes son los que menos consumen radio, pero al ser el grupo que sintoniza con mayor frecuencia los programas dirigidos a los adultos o al destinatario general se convierten en consumidores indirectos de lo que escuchan otros (padres o abuelos, por ejemplo) en función de sus intereses y preferencias. Es decir, la prevalencia de las generaciones mayores también se manifiesta en cuanto al empleo del medio. Por lo anterior puede inferirse que si bien los jóvenes no son en su mayoría la población que más escucha radio, pero lo hacen de manera indirecta al ser involucrados por los padres de familia para que también realicen el proceso de radio escucha con los estudiantes (Despaigne, 2012 citado por Badia y Costales, 2015).

La utilización de estos géneros radiales como material didáctico de manera auxiliar para facilitar el proceso del aprendizaje en la comprensión textual de los niños debe cumplir ciertas características para que sea eficaz, además de tener en cuenta algunas propiedades para que pueda llevarse a cabo en el proceso de enseñanza como se tenía previsto este debe ser: ser adecuado al 
asunto de la clase, de fácil aprehensión y manejo y estar en perfectas condiciones de funcionamiento.

Actualmente el problema de comprensión lectora presentado en los estudiantes de todos los grados y universitarios es muy común hoy en día. Siendo evidente la poca cultura de lectura que hay en la sociedad actual, la cual se ve reflejada en las pruebas externas que son aplicadas periódicamente por los gobiernos actuales (Instituto Colombiano para la Evaluación de la Educación Superior - ICFES, 2015).

Según el (Instituto Colombiano para la Evaluación de la Educación Superior - ICFES, 2015), el 90\% de los niños que se les aplico la prueba censal, estos informaron que el nivel de compresión lectora es apenas aceptable; teniendo un puntaje promedio de las tres (3) en tres 300 y 313 puntos, lo que representa una escala que va de 100 a 500 puntos. De igual manera, el nivel de desempeño que maneja el ICFES, menciona que este promedio mínimo no alcanza a llegar a ser satisfactorio (Bustamante, 2015).

A pesar de que, durante las dos últimas décadas, el gobierno ha hecho ingentes esfuerzos por mejorar la calidad de la educación en todo el país, introduciendo cambios en las concepciones de los contenidos curriculares, las evaluaciones y las prácticas pedagógicas. Estos cambios fueron plasmados en diferentes proyectos del Ministerio de Educación Nacional - MEN como la Ley General de Educación y el Decreto 1860 del mismo año que la reglamenta (Ley 115, 1994; Decreto 1860, 1994), el Sistema Nacional de la Evaluación de la Calidad (Pruebas SABER), resolución de indicadores de logros, lineamientos curriculares de lengua castellana, redi- seño de las evaluaciones de estado entre otros y pese a estas acciones tendientes a elevar la calidad del nivel educativo de los colombianos, con miras cumplir con el objetivo de ser el país más educado en el año 2025, los estudiantes de primaria y secundaria no muestran avances significativos en el desempeño de las pruebas en la región.

Cabe agregar, que, en la actualidad, existen propuesta metodológica están enfocadas en tomar varias propuestas en referencias a metodológica pedagogías basadas o fundadas en investigación, las cuales se desarrollan en diferentes contextos por el cual asumen variados caminos en coherencia con los paradigmas y corrientes en los cuales se inscriben.

Dentro de esta teoría basada en investigación se encuentra la enseñanza por descubrimiento y el Programa Ondas, con su propuesta de la Investigación como Estrategia Pedagógica (IEP), una perspectiva que se desarrolla en ese enfoque y toma elementos de la pedagogía critico-liberadora y del enfoque socio-cultural que abarca multiplicidad en las personas con el objetivo que la creatividad y poder de razonamiento sea consonó con los cambios actuales en materia investigativa,(Mejía y Manjarrés, 2010).

En los años ochenta fue promovida una propuesta metodológica que se desarrolló en Inglaterra y EE.UU, en el cual estuvo promovida por Jerome Bruner, y se expandió a España, donde fue acogida por algunos profesores de la Universidad de Valencia y la Universidad de Barcelona (Porlán,1995),este proceso fue iniciado con mecanismos semejantes y complementarios, donde a través de ellos se explicó con rigor el trabajo de grupos de infantes estrategias 
netamente investigativa, esto con el fin de lograr sus propios medios mediante la reflexión sobre ella, en ese sentido, por medios de este mecanismo se podrán descubrir una estructura con base a una realidad en los procesos, modelos y productos de la ciencia.

Entonces, los contenidos del proceso educativo se convierten en problemas, $y$ a partir de preguntas se estructura el currículo, donde los avances y el progreso pertinente a la práctica de una lectura como medio de aprendizaje, donde el estudiante determina la ampliación de una estructura enfocada en lenguaje y conocimiento guiado por el docente en cuestión con la focalización de llevar una planificación y los procedimientos para tal fin (Pozo, 2006).

Este aprendizaje está fundado sobre algunos principios que fundamentan los procesos de los contenidos:

1. Capacidad para resolver dificultades es el fin primordial de la educación.

2. El conocimiento verbal es la clave de cualquier transferencia.

3. El descubrimiento asegura la conservación del recuerdo.

4. El descubrimiento construye a cada estudiante, ser un ser con pensamiento crítico constructivo.

Es por ello, que la propuesta es construir una cultura ciudadana y democrática en ciencia, en donde por medio de esta herramienta que permite poder ser creativo y por ende cada estudiante se puede involucrar bajos criterios utilizando la práctica de la radio novela sobre un conocimiento bajo estas perspectivas. Este ejercicio pedagógico se considera fundamental para el avance del estudiante en pertinencia a una práctica para el aprendizaje, a través ideas de justas en el sentido educativo y justicia curricular, que trabaja por construir sociedades más justas y menos desiguales.

El punto de partida para buscar caminos alternativos en educación desde una perspectiva crítica se da a partir de un triple reconocimiento: Primero, la revolución técnica-científico permite ir más allá de una simple modernización de la educación que transforma unas relaciones de poder que controlan y dominan con propuestas pedagógicas y metodológicas inclusivas que muestren diseños específicos, más allá de la simple denuncia y crítica cónsonas con modernización en estos tiempos.

Por otro lado, la modernidad ha permitiendo convertir en una oportunidad más allá de una simple modernización de la educación interactuando para transformar las relaciones de poder que controlan y dominan con propuestas didácticas y metodológicas inclusivas que muestren diseños precisos, más allá de la simple denuncia y crítica general, lo cual lo convierte a actores que trabajan con el conocimiento, las tecnologías de la información y comunicación en una generación claves de los nuevos procesos de conformación de esta sociedad, alcanzando en los educadores todos los tipos de carácter que organiza y da forma a la base y fundamento de estas modificaciones sociales (Mejía, 2011; Herrera, Guerrero y Ramírez., 2018).

En ese sentido, las corrientes educativas enmarcan una acción que durante muchos años ha sido señalada como objetiva, y que no solo ha construido una forma de pensar basadas en la investigación, sino que también ha ayudado a crear formas de poder que en la sociedad que han servido para la vigilancia y la gestación en referencia a desigualdades, y en estos tiempos, 
nuevas formas de acumulación y dominación (Ortega, 2009).

En América latina, así como en grupos subalternos en el mundo, una concepción del trabajo educativo se caracteriza por ser una acción está enfocada con la intención es lograr generar conciencia crítica y dinámicas sociales que impulsen a los grupos construir formas de asociación y organización que los convierte en sujetos colectivos, constructores de su historia (Gadotti, 2003; Ramírez y Hugueth, 2017).

En los departamentos y municipios, objeto de la presente investigación los actores optan por desarrollar propuestas que constituyen redes (sociales, de actores e instituciones que hacen real esa búsqueda y se coordinan para concretarla en los diferentes territorios y espacios de sus respectivas localidades. Estas redes han reconocido la necesidad de transformar los procesos de la educación, de la relación entre los estudiantes que se mediatizan en estas prácticas están dinamizadas en un escenario de individuación, donde la integralidad de lo humano (razón, emoción, acción, intereses) debe ponerse en juego para reconocer que nos hacemos seres íntegros en todos los escenarios de nuestra colectividad y sus realidades.

Cabe decir que en la realidad se conoce una forma de transformarnos a nosotros mismos con un sentido de llevar las distintas formas de control pertinente a una dominación que traduce variadas manifestaciones de poder en esta sociedad, esto mediante políticas, económicas, sociales culturales basadas en un conocimiento de subjetividad de géneros en el ejercicio de la práctica educativa y pedagógica, en tal sentido, la misma busca construir un espíritu de emancipación humana que conduce al desarrollo de los procesos en su propia vida (Torres, 2007; Ramírez y Ampudia, 2018).

\section{Metodología}

La metodología del proyecto de investigación se realizó a partir de los recorridos de las trayectorias de indagación, basados en la integración de la IEP apoyada en TIC al aula, articulado al plan de estudios, desde las diferentes áreas se estructuraron temáticas relacionadas con la robótica educativa, la finalidad de este proceso investigativo, fue generar una trasformación social y educativa a través del aprendizaje en contexto, involucrando intereses, motivaciones y realidades de los estudiantes para generar conocimiento científico, donde se establecieron cinco (5) momentos que especifican en la ejecución y las metas trazadas para darle solución a la pregunta problema planteada

El eje de esta propuesta pedagógica está fundamentado en la idea en que esta sociedad existen saberes propios de una cultura propician en el intercambio de saberes, los cuales se negocian seguidamente con formas establecidas del conocimiento, es por ello que la investigación es parte fundamental dentro de los procesos como practica indispensable de la pedagogía, la cual busca involucrar de una forma sostenida los grupos infantiles y juveniles, mediante la relación de saberes y conocimiento como parte complementaria, a través de una propuesta metodológica que realiza el reconocimiento social de los actores, quienes ponen en juego esas diferentes concepciones, que podrán tramitar reconociendo la visibilidad de múltiples métodos investigativos, en coherencia con el tipo de problema. 


\section{Participantes}

La unidad de análisis estuvo conformada por cuarenta (40) estudiantes con edades que oscilaron entre los 7 y 9 años pertenecientes a los grados tercero y cuarto de primaria de la institución educativa Cerro Blanco, sede principal del Municipio de Zona Bananera, Magdalena. Cabe destacar que los participantes diligenciaron debidamente el consentimiento informado.

\section{Técnicas e instrumentos}

Esta investigación utiliza como fluentes de información múltiples herramientas que permiten recolectar insumos para tener de primera mano información proporcionada por actores intervinientes en esta investigación, siendo estos los estudiantes en primera instancia los cuales en el desarrollo de sus clases se implementó la Investigación del género radial tele-novelesco, que brinda aportes para que luego lo docentes puedan realizar el proceso de sistematización.

Se utilizaron como fuentes de información primarias: la observación, participante y de campo y entrevistas con estilo de encuesta cerrada a los estudiantes participantes de la Institución Educativa Departamental - LED; con la finalidad de identificar si este género da un aprendizaje significativo en los estudiantes durante el desarrollo de las actividades implementando la IEP.

\section{Procedimiento}

Se establecieron cinco (5) momentos que especifican en la ejecución y las metas trazadas para darle solución a la pregunta problema planteada.
A continuación, se presentan los diferentes momentos.

Momento (1): selección y análisis de la población sujeto de estudio.

Momento (2): integración de la IEP apoyada en TIC al aula de clases,

Momento (3): socialización de los resultados de las indagaciones de estudiantes en una feria institucional dirigida por los docentes a cargo.

Momento (4): análisis de los resultados de la implementación de la estrategia a través de la observación y diario de campo.

Momento (5): propagación y divulgación de los resultados.

Esta investigación utiliza como fuentes de información múltiples herramientas que permiten recolectar insumos para tener de primera mano información proporcionada por actores intervinientes en esta investigación, siendo estos los estudiantes en primera instancia los cuales en el desarrollo de sus clases se implementó la Investigación del género radial tele-novelesco, que brinda aportes para que luego lo docentes puedan realizar el proceso de sistematización.

Se utilizaron como fuentes de información primarias: la observación, participante y de campo y entrevistas con estilo de encuesta cerrada a los estudiantes participantes de la Institución Educativa Departamental - IED; con la finalidad de identificar si este género da un aprendizaje significativo en los estudiantes durante el desarrollo de las actividades implementando la IEP. 


\section{Resultados}

Con esta investigación los estudiantes de la Entidad Educativa Departamental mejoraron la práctica pedagógica utilizando la radionovela como herramienta clave para el desarrollo de capacidades con una comprensión coherente y por ende su desempeño académico, implementando esta estrategia novedosa aplicada como un gran puente y herramienta de utilidad en las clases impartidas; al igual que las redes sociales, las cuales no solo sirvieron para enviar lecturas que los estudiantes escuchaban sino para desarrollar talleres y compromisos en casa.

En ese orden de ideas, los anteriores resultados también sirvieron para que los padres, quienes en su mayoría tuvieron la oportunidad de conocer un poco sobre el proceso de la radionovela rescata la tradición oral, esta a su vez busca que, los estudiantes realicen un acompañamiento adecuado de estas mismas; y se implementa este proyecto de forma transversal ya que pueden ser utilizado en cualquier asignatura y grado de estudio, además permitan una relación con los valores, entrelazados con la ética, así pues, queden constituido por una serie de elementos que complementados entre si dan un sentido a la emisión de un programa.

La intención de esta práctica pedagógica, permitió llevar esta herramienta al aula de clases como una forma atractiva de enseñar, que además consistió en recordar a todos los involucrados dentro del referido entorno que las personas están dispuestas a modificar sus hábitos si se les demuestra que hay otras formas y mejores opciones para fomentar la cultura de la prevención que conlleva el desarrollo local, regional y nacional. En ese sentido, el estudiante pudo reconocer el contexto y la tradición oral que hace parte de una cultura en su región. Para ello se hizo una recolección de palabras que escucharon normalmente y eligieron las que creyeron pertinente a sus expectativas.

\section{Conclusiones}

Se llega a la conclusión que las investigaciones con estrategia pedagógica postula la existencia de saberes que se promueven una experiencia y actuaciones en la sociedad, permitiendo adquirir conciencia con elementos desde la tradición oral, que es trasmitida de generación en generación obviamente desde el narrador organiza de acuerdo a su convivencia mediante conocimientos que están dados en la esfera que implican un sinfín de actividades claves, las cuales favorecen aptitudes concretas y por lo general, complementarias de los estudiantes (Mignolo, 2003).

En efecto, estas también deben ser reconocidas por el ejercicio educativo, haciendo real la interculturalidad y la negociación cultural; este ejercicio debe darles cabida a las razones más allá de la "razón universal", para hacer visible el reconocimiento del otro y de lo otro, con la intencionalidad que permiten practicar la expresión oral, y acerca a los a los estudiantes a los valores comunicativos de elementos como la música, los efectos sonoros o el silencio, esto por medio de la elaboración de guiones de radio, que permiten desarrollar la capacidad de los estudiantes para sistematizar la información y dar coherencia desde el punto de vista radio fónico, al tiempo que incentivan la creatividad pertinente a los procesos inherente al entorno educativo. 
Desde ahí, lo que se construye en cualquier acción educativa son relaciones sociales, en las cuales se manifiestan bajo formas pedagógicas aquello que pensamos de la sociedad de manera libre y con igualdad de oportunidades para formular preferencias e igualdad de oportunidades para manifestar públicamente dichas preferencias (Valenzuela y Rochin, 2013). Es por eso, que el papel fundamental es la fundamentación pedagógica que sustenta el uso de la radio novela como práctica de los medios de comunicación audiovisual en nuestra sociedad y la consiguiente necesidad de favorecer una lectura crítica y creativa de los mismos, así como abrir la escuela a la realidad del entorno.

En este sentido, el papel del formando es activo y también forma a sus adultos acompañantes, a la vez que va aprendiendo, gestándose unas relaciones educativas desde lo diferente en la interculturalidad, transformando su rol tradicional y reconociéndose como aprendientes en el proceso (De Souza, 2001), la idea de aprendizaje es retomada también de este enfoque y reelaborada para Vigotsky: "El aprendizaje es un aspecto universal y necesario del proceso de desarrollo culturalmente organizado y específicamente humanos de las funciones psicológicas"; es decir, este, organizado pedagógicamente, y se convierte en desarrollo mental donde la comunicación ocupa un sentido en delimitar las posibilidades de acuerdo con las experiencias más relevantes emprendidas en un modelo de utilización de la radionovela con fines pedagógicos en el marco de los centros escolares.

\section{Referencias}

Badia, A. y Costales, Z. (2015). La audiencia radiofónica cubana en un contexto de cambios. Razón y Palabra, 19(92). 1-24. Recuperado de https:// w w w . r e d a l y c . o r g / pdf/1995/199543036013.pdf

Bustamante, N. (febrero 21, 2015). Niños colombianos pasan raspando en habilidad lectora. El tiempo. [Archivo]. Recuperado de http://www.eltiempo. com/archivo/documento/CMS 15283357

Cisneros, M., Olave, G. y Rojas, I. (2013). El lenguaje de la telenovela en la conducta lingüística de televidentes jóvenes: un estudio de caso. Perspectivas de la comunicación, 2(2). 7-17. Recuperado de http://publicacionescienciassociales.ufro.cl/index.php/ perspectivas/article/view/71/54

Cisneros, E. (2003). Breve Aproximación al estudio del lenguaje en la telenovela colombiana. Revista Litterae (12), 124-142. Recuperado de https://dialnet.unirioja.es/servlet/ articulo?codigo $=2745898$

De souza, J. (2001). Atualidade de Paulo Freire. Contribuição debate sobre a educação na diversidade cultural. Recife: Edições Bagaço.

Despaigne, Y. (2012). Los programas juveniles de las emisoras nacionales de radio. [Tesis Maestría]. Universidad de La Habana, La Habana, Cuba.

Gadotti, M. (2003). Perspectivas actuales de la educación. Buenos Aires: Siglo XXI.

Fals-Borda, O. (1984). Resistencia en el San Jorge. Historia doble de la Costa. Tomo III. Bogotá, D.C.: Carlos Valencia Editores. 
Haye, R. (1995). Hacia una nueva radio. Argentina: Páidos.

Herrera, B., Guerrero, H. y Ramírez, R. (2018). Investigación como estrategia pedagógica: Una mirada desde la educación, escuela y transformación de la comunidad global. Barranquilla: Editorial Universitaria de la Costa-Educosta.

Mariño, G. (2010). El diálogo en la educación de jóvenes y adultos. Dos propuestas pedagógicas para implementarlo. En, El taller dialógico/la recuperación de experiencias laborales. Bogotá, D.C.: OEI. Recuperado de http://www.germanmarino.com / p hoc adown loadpap/ DIALOGO\%20EN\%20LA\%20EDUCACIN\%20JVENES\%20ADULTOS.pdf

Mejía, M. y Manjarrés M. (2010). Las pedagogías fundadas en la investigación. Búsquedas en la reconfiguración de la educación. +Revista Internacional Magisterio. 42(1). 1626. Recuperado de http://eduteka. icesi.edu.co/articulos/PedagogiasInvestigacion

Mejía, M. (2011). Las escuelas de la globalización. El conflicto por su reconfiguración. Bogotá, D.C.: Desde Abajo.

Mignolo, W. (2003). Capitalismo y geopolítica del conocimiento. Buenos Aires: Paidós.

Ortega, P. (2009). Sujetos y prácticas de la pedagogía crítica. Bogotá, D.C.: El Búho.

Porlán, R. (1995). Constructivismo y escuela: hacia un modelo de enseñanza aprendizaje basado en la investigación. Sevilla: Diada.

Pozo, J. y. (2006). Aprender y enseñar ciencia. Madrid: Morata.
Ramírez, R. y Hugueth, A. (2017). Modelo de comunicación productiva para las organizaciones de salud pública en Venezuela. Opción. 33(83). 305336. Recuperado de http://produccioncientificaluz.org/index.php/ opcion/article/view/23118/23159

Ramírez, R. y Ampudia, D. (2018). Factores de Competitividad Empresarial en el Sector Comercial. Revista Electrónica de Ciencia y Tecnología del Instituto Universitario de Tecnología de Maracaibo (RECITIUTM). 4(1). 16-32. Recuperado de http://recitiutm.iutm.edu.ve/index.php/recitiutm/article/view/130

República de Colombia. Instituto Colombiano para la Evaluación de la Educación Superior - ICFES. (2015). SABER 11. Resultados nacionales 2011- 2014 Icfes. Recuperado de h t t p s : / / w w w. i c f e s. g o v. co\% 2 Fdocman\%2Finstituciones-educativas-y-secretarias $\% 2 \mathrm{Fsaber}$ $11 \% 2 \mathrm{~F}$ in for mes - d e - s a ber 11\%2F2073-resultados-nacionalessaber-11-2011-2014\%2Ffile\%3Fforcedownload\%3D1\&usg=AOvVaw34WA BEI1ekdvFqRQZDe93M

República de Colombia. Congreso de la República. (febrero 8 de 1994). Por la cual se expide la ley general de educación. [Ley 115]. DO: 41.214. Recuperado de https://www.mineducacion. gov.co/1621/articles-85906_archivo_ pdf.pdf

República de Colombia. Presidencia de la República. (agosto 5 de 1994). Por el cual se reglamenta parcialmente la Ley 115 de 1994, en los aspectos pedagógicos y organizativos generales. [Decreto 1860]. DO: 41.473. Recuperado de https://www.mineducacion. gov.co/1621/articles-172061_archivo_pdf_decreto1860_94.pdf 
Torres, A. (2007). La educación popular. Trayectoria y actualidad. Bogotá, D.C.: El Búho.

Trujillo, P. (2013). La radionovela como una práctica didáctica para prevenir las adicciones. [Tesis]. Universidad Autónoma de México. México D.F., México. Recuperado de http://132.248.9.195/ ptd2014/enero/0707411/0707411.pdf
Valenzuela, G. y Rochin, L. (2013). Libertad de expresión y su impacto en la democracia. Juridicas CUC, 9(1), 89-100. Recuperado de https://revistascientificas.cuc. edu.co/juridicascuc/article/view/441/ pdf_27

Vilches, L. (2003). La televisión. Los efectos del bien y del mal. Barcelona: Paidós. 